FORMATION Formation emploi

Revue française de sciences sociales

122 | Avril-Juin 2013

Relever les défis de la Validation des Acquis de l'Expérience (VAE)

\title{
Le travail des jurys de VAE ou la mise en scène de l'expérience
}

The work of the Accreditation of Prior Learning (APL) juries or a representation of the candidates 's experience

Die Arbeit der VAE-Prüfungsausschüsse oder die Inszenierung der Berufserfahrung

El trabajo de los jurados de la Validación de la Experiencia Adquirida o la puesta en escena de la experiencia

\section{Emmanuelle Auras}

\section{OpenEdition}

Journals

Édition électronique

URL : http://journals.openedition.org/formationemploi/3990

DOI : 10.4000/formationemploi.3990

ISSN : 2107-0946

Éditeur

La Documentation française

Édition imprimée

Date de publication : 30 juin 2013

ISSN : 0759-6340

Référence électronique

Emmanuelle Auras, "Le travail des jurys de VAE ou la mise en scène de l'expérience », Formation emploi [En ligne], 122 | Avril-Juin 2013, mis en ligne le 16 juillet 2013, consulté le 30 octobre 2020 URL : http://journals.openedition.org/formationemploi/3990 ; DOI : https://doi.org/10.4000/ formationemploi.3990

(c) Tous droits réservés 


\section{Le travail des jurys de VAE ou la mise en scène de l'expérience}

Emmanuelle Auras Ingénieur de recherches, sociologie (GRESCO EA 38-15), directrice du service de formation continue de l'université de Poitiers

Résumé

\section{Le travail des jurys de vae ou la mise en scène de l'expérience}

Une observation des jurys de VAE (validation des acquis de l'expérience) et une analogie avec la métaphore théâtrale d'Erving Goffman montrent que la décision s'appuie sur une mise en scène de l'expérience du candidat, qui doit coïncider avec les normes universitaires et professionnelAles des jurys. À cette fin, le candidat choisit les éléments de son parcours concordants avec leurs attentes implicites et explicites. Le travail du jury s'oriente alors vers la vérification de l'authenticité de la représentation, à l'aide d'indices relevés dans le dossier et au cours de l'entretien.

Mots clés : Validation des acquis de l'expérience

Abstract

The work of the Accreditation of Prior Learning (APL) juries or a representation of the candidates 's expérience

An observation of the APL's juries and an analogy with the dramaturgic metaphor of Erving Goffman show that the decision relies on a representation of the candidate's experience, which has to coincide with the academic and professional standards of the juries. To this end, the candidate chooses the elements of his/her career corresponding to their implicit and explicit expectations. The work of the jury turns then to the check of the representation's authenticity, by finding indications both in the file and during the interview.

Key words : validation of acquired skills

Journal of Economic Literature: I 29

Traduction : Auteur 
Le fonctionnement des jurys de validation des acquis (VAE) est régulièrement interrogé : sociologues, psychologues et didacticiens fournissent une riche production scientifique pour élucider la question clé de la VAE : comment traduire des parcours d'expériences en éléments de diplômes ? Ropé (2005) montre que ceux qu'elle nomme les agents de la VAE débattent de la manière de présenter l'expérience aux membres du jury, opposant les tenants de la rationalisation de la décision qui construisent des outils de mesure et ceux qui mettent en œuvre ad hoc des processus empiriques de mise en évidence de preuves tangibles.

Cette question s'élucide en partie dans l'espace clos du jury par des séances d'observation. Le jury peut être considéré comme une activité de travail comme une autre : Prot (2003) montre ainsi comment le référentiel devient une norme collective et s'enrichit au cours de son usage en VAE. Mayen et Mayeux (2009) décryptent le contexte dans lequel les jurys doivent se débrouiller (buts, obstacles, ressources, instruments) : l'utilisation des seuls référentiels institués est présentée comme nécessaire mais insuffisante pour cette activité. Ils opèrent un ajustement de ces référentiels en mobilisant une multitude de ressources constituées par les univers des candidats et les cadres de référence des jurys. Dans une démarche comparable, Mayen et Métral (2009) observent un parallèle avec les modalités de décision de jurys d'admission en écoles d'ingénieurs : ils ajustent leur modalité d'évaluation autour de critères essentiels communs à tous les candidats. Ils découvrent des indicateurs à la lecture du dossier, les discutent ensemble, les confrontent au discours du candidat. Ils comparent son profil à celui de professionnels ou d'étudiants rencontrés auparavant. Ils utilisent les ressources à leur disposition pour résoudre les problèmes que leur pose a priori l'évaluation des acquis de l'expérience : fiches $\mathrm{RNCP}^{1}$, définitions de l'ingénieur, expériences passées, échanges entre membres.

Un autre angle de vue met au jour une norme indigène de validation dans les jurys universitaires : Auras (2004) montre une typologie des jurys en fonction de leur manière d'interpréter la loi et de s'approprier l'acte de validation.

Le dossier comme objet d'étude contribue à la compréhension des décisions et Géhin (2011) l'aborde sous l'angle des acquis de l'engagement. En décrivant les logiques de présentation de ces acquis, il observe leur prise en compte par le jury. Des stratégies mises en œuvre dans les dossiers apparaissent plus efficaces que d'autres : la conversion quasi automatique des expériences militantes en savoirs professionnels ne semble pas convaincre les jurys ; en revanche, l'euphémisation consistant à réduire les activités d'engagement à des dimensions techniques, gommant les aspects politiques, remporte un meilleur succès.

1. Répertoire National des Certifications Professionnelles. 
Ce panorama confirme que loin d'être mécaniste, l'activité des jurys est socialement construite, s'adapte aux contextes, s'ancre dans l'ensemble des ressources à leur disposition, permettant la fabrication d'une norme à la fois stable et nuancée par des ajustements continus.

À l'université, la VAE apparaît complexe à mettre en œuvre : comment traduire des parcours d'expérience, par définition non linéaires, en contenus de formation sans appui sur un référentiel de compétences?

Nous faisons l'hypothèse avec Vial et Mencacci (2004) que le diplôme ne se limite pas aux savoirs transmis formellement, mais que la formation universitaire propose une socialisation intellectuelle : des éléments implicites, invisibles dans les référentiels, sont pris en compte en formation initiale comme en VAE. À côté de la description des métiers et des diplômes, les auteurs identifient un référentiel de formation, permettant à la fois de s'orienter vers la culture de métier et de se familiariser avec la démarche scientifique. Pour eux, ce " processus de professionnalisation " constitue une originalité de l'université et lui offre une légitimité : développement de la fonction critique, acquisition de méthodes et de références savantes. En prolongeant ces travaux, il est pertinent d'approfondir le rôle de la culture universitaire et des attentes symboliques des jurys.

Deux questions se posent : comment les jurys réalisent-ils cette mesure de l'expérience en mobilisant des ressources limitées comparées à celles dont ils disposent pour évaluer un étudiant ? Où trouvent-ils les indicateurs de la présence des implicites du diplôme, les compétences transversales attendues? Nous faisons l'hypothèse que la décision se prend aussi sur des éléments symboliques, à travers un processus d'interactions entre les membres des jurys et le candidat. Elle se joue « ici et maintenant » mais s'appuie sur des cadres de références extérieurs au temps du jury. Puisque la VAE ne se trouve pas toute entière dans des composants objectivables construits a priori, nous la commenterons sous l'angle microsocial du «temps du jury ». Elle est la production d'un groupe restreint, dans une contrainte spatiotemporelle particulière : la lecture d'un dossier, le temps de l'entretien.

Un choix méthodologique s'est imposé pour saisir la construction de la réalité sociale des jurys de VAE : un terrain circonscrit propice à l'application d'une enquête compréhensive ; l'analogie avec les travaux du sociologue Ervin Goffman. Dans une première partie, nous justifierons ces choix, décrirons le terrain d'enquête et déclinerons la grille de lecture inspirée des travaux de Goffman. Dans une seconde partie, nous présenterons les résultats et montrerons comment les membres des jurys opèrent en situation pratique, par comparaison avec des situations antérieures, en quête d'une proximité culturelle avec le candidat, mobilisant à cette fin le dossier et l'entretien, deux manières pour le candidat de mettre en scène son expérience. 


\section{Observation participante et métaphore théâtrale}

Ce travail s'appuie sur une observation participante, propice à déceler des éléments implicites. Il s'articule autour de la métaphore théâtrale de Goffman afin de réintroduire une distance avec une réalité professionnelle familière.

\subsection{Des jurys pluridisciplinaires expérimentés en VAE}

L'université dans laquelle l'enquête s'est déroulée est de taille moyenne, pluridisciplinaire. Une organisation mixte de la VAE est caractérisée par la centralisation de l'accompagnement dans le service de formation continue et une décentralisation des jurys dans les UFR (unités de formation par la recherche). Cette université se classe dans les cinq premières en France en termes de dossiers de VAE déposés en 2009². Ceci peut en partie s'expliquer par la présence d'un dispositif de VAP (validation des acquis professionnels) rôdé depuis 1995, encadré par des procédures construites collectivement et une ingénierie de conseil individuel bien établie. Comme ailleurs, les membres des jurys sont des enseignants, des enseignants-chercheurs et des professionnels. Un noyau permanent est constitué d'un président (la plupart du temps un professeur) et de vice-présidents garantissant la mémoire et la continuité dans les pratiques, les autres membres étant associés en fonction des dossiers.

L'auteur, cadre du service depuis 2000, a participé à de nombreux jurys. Cet article se fonde sur une monographie de la formation continue dans cette université, réalisée de 2002 à $2011^{3}$, intégrant l'observation de 16 séances de jurys statuant sur des licences professionnelles et des diplômes dits généralistes (sciences humaines, lettres et langues).

2. Note d'information 11-03-2010, DEPP, ministère de l'Education nationale.

3. Auras E., De paradoxes en compromis l'intégration de la formation continue à l'université, Thèse de doctorat en sociologie, Poitiers, 2011. 


\section{Encadré 1}

\section{Jurys observés}

IUT (Institut universitaire technologique) : licences professionnelles « Hygiène et Sécurité » (3), «Commerce et Distribution » (2), « Banque Assurances » (4), « Gestion des Ressources Humaines » (1), « Logistique et Transports Internationaux » (3).

- Sciences humaines et arts : licence de psychologie (5) ; master d'ergonomie et psychologie du travail (2),

- Institut de la Communication et de l'Information (ICOMTEC) : I'IUP (institut universitaire professionnalisé) «Information et Communication des Entreprises » (3),

- Sciences du sport : licences professionnelles «Animation des activités sportives » (3), « Management du sport » (2), «Activités sportives adaptées » (1),

- Lettres et langues : licence langues étrangères appliquées, (1) licence langues, littérature civilisations étrangères(3), licence de lettres modernes (3).

\section{Grille d'analyse des notes, inspirée de Goffman :}

- Références à d'autres sphères (jurys de Validation des acquis professionnels ou de l'expérience, commissions, soutenances, connaissance des métiers) ;

- Indices d'une connivence culturelle avec le candidat (attitude, réaction des membres, ton de l'oral, indicateurs du rapport au savoir, distance/familiarité).

- Eléments perturbateurs (indices de contradictions entre le dossier et l'entretien, référence à des lectures jugées «profanes », expériences jugées disqualifiantes);

- Façade du candidat (manière de se mouvoir, signes non verbaux, assurance ou doute à l'oral, «ton » du dossier) ; réactions des jurys (remarques sur la problématisation, sur l'accent mis sur les détails, les techniques) ;

- Répartition des rôles entre les membres (jugement disciplinaire, niveau général, aspects professionnels).

\section{Entretiens approfondis enregistrés :}

- Cinq candidats, six membres universitaires et quatre accompagnateurs ;

- Dans le bureau des enseignants-chercheurs, au service de VAE pour les candidats et les accompagnateurs ;

- Portant sur : l'évocation des attentes vis-à-vis des candidats, comparaison avec les étudiants en réussite, difficultés d'évaluation (jurys) ; lecture des attentes des jurys, observations personnelles sur la décision (accompagnatrices) ; sentiment après la séance de jury et l'annonce de la décision (candidats).

$\left({ }^{*}\right)$ : Une forme descriptive ou analytique ; un style littéraire, technique ou scientifique ; assurance, valorisation des compétences acquises, versus doute et minimisation des qualités professionnelles. 


\subsection{Une approche ethnographique : saisir le non formel de la décision}

La description d'un monde social restreint fait référence à la tradition ethnographique. Cette enquête peut être qualifiée d'observation participante, puisque l'enquêteur est membre de la situation par son statut professionnel. Dans cette posture "observation participante complète par opportunité ", il met à profit une place déjà acquise (Adler P. et P., 1987, cité par Soulé, 2007, p. 3).

Se pose alors la question de la distance entre le chercheur et son objet. Portée par les sociologies anglo-saxonnes issues de l'Ecole de Chicago, la proximité est privilégiée en ethnométhodologie, une partie de l'interactionnisme et dans l'enquête ethnographique. Les chercheurs inscrits dans ces courants s'intéressent à des objets microsociaux et recherchent la proximité entre l'enquêteur et son terrain pour entrer dans les pensées de l'enquêté. " $L a$ présence sur le terrain n'est pas seulement destinée à décrypter un système social de l'intérieur; elle vise à comprendre ce que c'est qu'être un acteur de ce terrain" (Soulé, 2007, p.8).

Pierre Bourdieu a lui aussi utilisé la proximité dans la Misère du Monde (1993, p. 1392), considérant que "le rêve positiviste d'une parfaite innocence épistémologique " ne sera pas atteint, signifiant ainsi que la proximité n'est pas plus risquée que la méthode hypothético-déductive. Afin d'éviter les distorsions liées à la situation d'enquête, il préconise une posture de réflexivité, permettant de s'alerter, par exemple, sur le risque de mettre à l'écart des indices fondamentaux pour la compréhension de mécanismes sociaux en jeu, considérés comme des allants-de-soi.

Diaz (2005, cité par Soulé, 2007, p. 3,) résume ainsi le dilemme : "Comment concilier la nécessité méthodologique de l'implication dans la vie d'un groupe avec le recul et la mise en perspective nécessaires au rôle de chercheur "? Il nous fallait alors trouver une manière d'assurer des allers et retours entre un terrain familier et un apport extérieur susceptible de le mettre à distance.

\subsection{La métaphore théâtrale pour une rupture du regard}

La référence aux travaux de Erving Goffman, en particulier "La présentation de soi ", (1973) comme appui théorique indépendant de l'objet observé, répond à ce dilemme.

Selon Goffman, le sociologue dispose du même sens commun que tout acteur social et ne doit pas chercher à rompre avec lui. Néanmoins, le savoir implicite relevant de routines inconscientes déployées par les participants d'une interaction résiste à l'analyse. Afin de dépasser cette difficulté, il fait apparaître ce qui semble aller de soi par l'adoption d'une "perspective par incongruité ». Il utilise pour cela un appareil descriptif a priori étranger à l'objet étudié pour traduire les interactions quotidiennes, comme la métaphore théâtrale : "Revoir les objets que l'on n'avait pas remarqués, les rendre anthropologiquement étranges nous permet de les observer d'un point de vue détaché de l'attitude naturelle " (Watson, 1999, p. 87.) Cette technique se prête à notre enquête, la posture d'observation à travers la 
métaphore théâtrale favorisant un regard neuf porté sur les scènes familières de jury en " mettant l'accent sur des traits formels communs à des situations quotidiennes dont on ne perçoit pas les similitudes", (Bonicco, 2006, p. 45).

Ainsi, la métaphore de La présentation de soi (Goffman, 1973) répond à la question de l'élucidation du non-formel d'un jury de VAE. Pour Goffman en effet, dès qu'un individu est en présence d'autres personnes, son comportement dégage une signification qu'elles interprètent : il transmet une "impression du moi ", par une attitude, des mots, autant d'indices pour se faire une idée de ce qu'il est, ce qu'il fait. Néanmoins, les acteurs et leur public ne partent pas de rien pour produire cette représentation et utilisent des références d'ordre général qui dépassent la relation immédiate. On peut transposer ces affirmations au dossier et à l'entretien de VAE, autant de manières de se présenter à ce jury dont on attend la reconnaissance.

\section{La fabrique de la validation}

Le jury de VAE s'apparente à une représentation : "la totalité de l'activité d'un acteur qui se déroule dans un laps de temps caractérisé par la présence continuelle de l'acteur en face d'un ensemble déterminé d'observateurs influencés par cette activité " (Goffman, 1973, p. 23). Des données spatio-temporelles caractérisent le temps du jury : la scène est jouée par cinq à huit personnes, dans un lieu unique, en huis-clos. La décision est prise rapidement sur la base d'un document qui résume la vie d'une personne inconnue et lors d'un bref entretien au cours duquel elle doit convaincre. Dans un champ aussi étroit, il apparaît que les attributs attachés à l'expérience du candidat sont insuffisants pour nourrir le débat.

Dans cette partie, nous allons montrer que les jurys utilisent tout ce qu'ils ont à disposition pour se construire "une définition de la situation " et identifier qui se trouve vraiment en face d'eux. De son côté, le candidat, avec l'aide de l'accompagnateur, cherche à renvoyer une image concordante avec les valeurs admises par l'institution.

Nous observerons une norme implicite que les membres élaborent autour des codes culturels collectifs afin de se construire une opinion, en limitant les conflits et les risques d'erreurs. Pour atteindre une bonne définition de la situation, ils s'appuient sur des informations extérieures au temps du jury, recherchent des indices de la proximité avec les fondamentaux de la culture universitaire et de la norme professionnelle. Ils s'appuient sur deux supports, le dossier et l'entretien, pour vérifier que le candidat possède les attributs attendus. 


\subsection{Les références extérieures au temps du jury}

La "définition initiale de la situation" (Goffman, 1973, p. 13) est fondée en partie sur le passé : les membres des jurys n'ancrent pas uniquement leur décision «ici et maintenant "; ils saisissent des éléments qui ne sont pas des attributs personnels du candidat, mais se rapportent à des situations d'ordre général. Ils réactivent des références à d'autres sphères : connaissance des métiers, entreprises fréquentées. Dans une majorité de jurys, une partie de la décision se prend par système d'analogie avec d'autres instances d'évaluation (commission d'équivalence, soutenance), des jurys de VAE précédents, des expériences de réussite de personnes en formation continue. Trois cas illustrent la référence analogique :

- Le jury de psychologie doit faire face à une situation complexe. Il est difficile de trouver dans une expérience l'équivalent de la connaissance théorique et de la socialisation professionnelle développées pendant la formation initiale de psychologue. Ses membres utilisent alors des systèmes de correspondance édifiés d'un jury à l'autre, sans construction formelle, débouchant sur des "types " de validation. "Tu te souviens, de Mme X qui était cadre dans cet hôpital ? On peut au moins lui valider la même chose!" (maître de conférences, psychologie sociale, membre permanent). Ce réflexe est renforcé par le fait que des personnes aux parcours similaires se présentent régulièrement devant ce jury (infirmière psychiatrique, professeur des écoles, assistante sociale).

- Le jury de lettres et langues, quant à lui, convoque le passé d'une manière plus formelle, même s'il utilise comme le précédent la mémoire et les déclarations des membres permanents, sans référence écrite : il fait appel au "répertoire des séances antérieures" (Mayen, Mayeux, 2009) : "Ça ne vous rappelle pas cette personne qui avait créé son cabinet de traduction, à Marseille? Nous n'avions pas tout validé! ", (maittre de conférences en langues littérature et civilisation, membre permanent), "reprenons le tableau des décisions, si on reprend la formatrice à l'AFPA, elle n'est pas loin, formations continues, et a vécu 6 ans à Boston, on n'est pas loin, on a validé tout le diplôme, mais là, sa connaissance de la littérature... trop faible ", (même personne, autre séance).

- Le jury de la licence hygiène et sécurité des biens et des personnes, enfin, étudie fréquemment des demandes de pompiers souhaitant intégrer la sécurité civile. La décision semble se dégager d'elle-même, construite collectivement au fil des séances, sans pour autant être formellement présentée et explicitée : il manque aux candidats quelques expériences significatives pour ce diplôme (par exemple la connaissance des services départementaux d'incendie et de sécurité, un niveau de responsabilité, la gestion d'une cellule de crise) pour lesquels ce jury préconise une gamme de formations complémentaires permettant la validation totale du diplôme (par exemple un module spécifique portant sur les SDIS, un rapport sur un des domaines manquants). La décision se fonde au premier plan sur ces critères enregistrés d'une manière quasi mécanique, et au second plan sur des facteurs individuels intervenant à la marge sur des validations supérieures. 
Ces trois jurys façonnent une représentation de la situation à partir de la somme des décisions antérieures, ou sur le souvenir qu'ils en ont. Aucun d'entre eux n'utilise formellement un tableau de références reprenant les décisions de jurys passés. La construction de cette référence collective se façonne au fil des échanges, des réunions de jurys, des interactions entre les membres, comme une règle implicite, légitimée par tous...

Cet appel au passé se manifeste aussi dans l'utilisation de stéréotypes portant sur des métiers enracinés dans notre patrimoine collectif. En sciences humaines, les candidats professeurs des écoles, éducateurs spécialisés ou assistants sociaux obtiennent des validations équivalentes ${ }^{4}$. Le jury ne statue pas sur la manière dont la personne habite sa profession, mais sur la profession elle-même. Chaque candidat devient alors le représentant d'un collectif qui le dépasse : "Un instituteur spécialisé, d'habitude, non, c'est une entrée en licence avec dispenses des modules $X$ et $Y$... ?" (maitre de conférences en psychologie sociale, membre non permanent). Cette référence collective malmène les affirmations des pionniers de la VAE : "Il s'agit de réaliser une évaluation à chaque fois spécifique pour s'adapter à la réalité de l'expérience, au particularisme de chaque parcours et aux modes d'apprentissage de chacun [...]” (Feutrie, 2004, p. 100).

\section{2 À la recherche des preuves partagées}

La "preuve " est une notion centrale en VAE : comment le candidat peut-il mettre en évidence des preuves de ses compétences ? Comment le jury les sélectionne-t-il parmi celles qui émergent formellement ou implicitement ? Elles n'apparaissent pas toutes dans les descriptions d'activité : parfois invisibles pour l'observateur, les jurys les repèrent sans hésiter. Fruits de la culture académique et des cultures professionnelles, les indices partagés sont générés au fil du travail de VAE, à l'articulation des savoirs savants et des savoirs d'actions. Ils se manifestent sous deux formes principales, la recherche d'indices de connivence partagés et la chasse aux indices dissonants. Une absence d'éléments de jugement peut aussi nuire à la décision, comme nous allons le montrer dans les paragraphes suivants.

En sciences du sport, la "connaissance du milieu sportif» est immédiatement saisie comme trace de l'imprégnation des codes et de la morale professionnelle. Cette notion floue se manifeste dans un accord tacite entre le professionnel et le reste du jury. Elle s'enracine dans un ensemble d'impressions dégagées par le récit du candidat.

À l'IUT, pour la licence "Commerce et Distribution », la pratique commerciale ne suffit pas, elle doit s'appuyer sur une connaissance des enjeux stratégiques du secteur. La fréquentation de plusieurs entreprises (du petit commerce à la grande distribution) est appré-

4. Par exemple, pour un instituteur titulaire d'une licence dans une autre discipline, une validation très partielle, portant sur des modules transversaux, professionnels, ou en langues. La délivrance du diplôme nécessite une reprise d'étude longue. 
ciée, la référence à une enseigne au mode de management connu des membres est une garantie. "Ils ne sont pas choisis comme gérants par hasard, chez X, ils ont des formations régulières, et les séminaires annuels les mettent au parfum sur la stratégie commerciale ". "Moi je veux bien mais sa fonction ne lui a pas permis de simpliquer, il ne connait visiblement pas le contexte du commerce du textile. Il ne connait que le discours offciel des franchises chez $X$ " (Professionnel, membre non permanent).

Ces débats dont le sens est inaccessible pour les accompagnateurs présents en jury non familiers du métier concerné sont constitutifs d'une définition de la situation. Au cours d'un jury de licence "Banque-Assurance ", enseignants et professionnels se consultent pour trouver des indices de "la fibre commerciale » dans deux dossiers a priori très proches. "Le dernier avait la fibre commerciale! Tu vois bien, la différence, c'est que celui-ci ne l'a pas" (professionnel, membre non permanent du jury échangeant avec un maître de conférences en sciences de gestion, membre permanent). Le jury, expert de la culture du métier, sait quoi chercher : un candidat évoque les stratégies de vente ; un autre affirme sa connaissance des produits financiers et ses "qualités relationnelles".

Au cours d'un entretien, un membre extérieur du jury précise qu'un professionnel de la banque doit connaitre l'importance de la fonction commerciale et doit savoir exposer une stratégie correspondante : le deuxième candidat a manifesté une lacune, élément discordant dans la définition de la situation.

Les dissonances manifestées par le candidat participent aussi à l'élaboration du travail des jurys : des indices renforcent la bonne impression qui se dégage de lui, d'autres la dégradent face au rôle attendu. En opérant un choix dans les facettes de sa vie, il peut mettre en avant l'élément dissonant. L'exemple le plus significatif s'observe en sciences humaines, dont la proximité entre les vocabulaires de sens commun et scientifique favorise les confusions. Un candidat au master de psychologie cite ainsi la revue Psychologie magazine; un autre décrit ses formations en "graphologie "; des allusions à la " PNL » sont également stigmatisantes. Non seulement ces références ne constituent pas des preuves pertinentes, mais elles dégradent l'image du candidat. Il a manqué de discernement, indiquant un écart avec la culture disciplinaire et la déontologie attachée au métier de psychologue.

Au cours des jurys du DUT (diplôme universitaire de technologie) " Commerce et Distribution ", la description d'une carrière dans une seule enseigne est le signal d'une expérience insuffisante. Le candidat est devenu expert de son mode de fonctionnement, de sa stratégie, ce qui l'éloigne d'une connaissance globale du monde commercial : le parcours est jugé trop partiel au regard des exigences généralistes du diplôme.

Dans un autre registre, parfois le candidat ne montre rien, ni dans l'attitude ni dans le récit, permettant de fournir des informations suffisantes pour orienter la décision. Les membres du jury cherchent en vain à repérer quelle est sa position dans l'entreprise, dans l'échelle des responsabilités. En relisant le dossier, ils émettent des doutes, comme ce jury de licence "Gestion des Entreprises et des Administrations " : "Qu'est-ce qu'elle fait vrai- 
ment dans ce service? On ne voit pas bien ce qu'elle faisait.» (professionnel, membre non permanent). "Elle veut faire quoi, se mettre à son compte ?"; ou celui de l'IUP «Information et Communication des Entreprises": "Je me souviens bien de cette personne! Un drôle de sentiment, il n'y avait rien! Ça ne faisait pas réel! Et pourtant il en a fait!" (ndl: des emplois hôteliers), (maitre de conférences en sociologie, membre permanent).

Plus grave encore, le jury peut craindre "la représentation frauduleuse" (Goffman, 1973, p. 61) : un individu possédant certaines caractéristiques sociales est normalement en droit d'attendre qu'on l'estime à ce niveau, mais l'interlocuteur exige en retour qu'il soit réellement "ce quill prétend être" (Goffman, 1973, p. 19). Ainsi, l'image du candidat se trouble pour ce jury de licence professionnelle "Management des Echanges Internationaux ": "Un poste honorifique, c'est tout, en fait il n'est pas à un niveau d'organisation, dans le service, c'est un technicien " (membre professionnel, non permanent,) : il y a discordance.

Un décalage entre l'écrit et l'oral en est aussi la manifestation. Au cours d'un jury en "Information et Communication des Entreprises ", le dossier d'une candidate mentionnant une carrière de gestion hôtelière paraissait solide, mais à l'oral elle n'a pas été en mesure de répondre aux questions : «En fait, cétait du vent» (maitre de conférences en sciences de l'information et de la communication, IUP, membre permanent).

Les jurys cherchent à voir si le candidat les trompe : lorsqu'ils découvrent qu'il « n’avait pas le droit de jouer le rôle qu'il jouait" (Goffman, 1973, p. 62), survalorisait ses responsabilités, ses connaissances, il peut "perdre définitivement sa réputation", (Goffman, 1973, p. 61) et a peu de chances d'obtenir une validation satisfaisante. Cette attente se révèle pour le jury de l'IUT dans la "grille de positionnement " décrite par Bellini (2011), dans laquelle le candidat auto-évalue ses compétences. En écoutant le jury, il semble que la grille serve autant à vérifier la sincérité du candidat qu’à faciliter la décision : elle participe à la définition collective de la situation et l'informe sur un attribut fondamental du demandeur : sa sincérité.

\subsection{Les supports de la définition : I'entretien et le dossier}

$\mathrm{Au}$ cours d'un entretien et dans un dossier, le candidat met en scène une "réalisation dramatique " (Goffman, 1973, p. 36) pour exprimer la qualité qu'il revendique. Les membres du jury font l'hypothèse qu'il cherche à se présenter sous son meilleur jour et ils doivent deviner ses intentions. Ils peuvent utiliser " la façade ${ }^{5}$ » du candidat, qu'il doit ajuster entre culture professionnelle et culture universitaire.

5. "Signes distinctifs de la fonction ou du grade, le vêtement, le sexe, la façon de parler, les mimiques, les comportements gestuels» (Goffman, 1973 p. 30). 


\subsubsection{L'entretien}

L'entretien se déroule différemment en fonction du caractère plus ou moins professionnel des filières. À l'IUT, il est orchestré par un style de "relations professionnelles ", proche d'un entretien de recrutement, (présentation du parcours, motivations, questions-réponses portant sur les fonctions) ; les jurys de sciences humaines, sciences du sport ou lettres et langues quant à eux restent attachés à la tradition scolastique et reproduisent le modèle de la soutenance (demande de prise de recul, recherche de la démarche d'analyse, interactions comparables à celles entre un étudiant et un enseignant). Les deux formes sont susceptibles de déstabiliser le candidat s'il n’en possède pas les règles du jeu : le ton utilisé peut être mal perçu par celui qui ne connaît pas le rituel.

Certains candidats repartent remplis d'amertume. D'autres sont surpris d'apprendre une décision positive, comme cette jeune dirigeante d'entreprise : au cours de l'entretien, des questions portent sur des thèmes professionnels (management, charges sociales) et ses réponses sont contestées par des membres du jury qui manifestent pourtant au départ une admiration pour sa maturité dans son rôle de jeune chef d'entreprise. Quelle ne fut pas sa surprise à l'annonce de la décision positive : "J'étais sûre que cétait négatif! J'étais dégoûtée... " (Entretien avec la candidate).

D'autres manifestent un comportement dissonant par rapport à l'attitude attendue. C'est le cas d'une candidate titulaire d'un BTS (brevet de technicien supérieur) " Marketing » et d'une courte carrière dans un service de communication. Elle se comporte selon un style très typé, utilisant un vocabulaire familier ("le service de com», "je suis créative ") marqué par sa culture professionnelle ("j'ai fait des flyers avec les graphistes, je suis plutôt douée pour faire de la $P A O »)$. Son attitude désinvolte était en dissonance avec la culture académique de ce diplôme. La contrariété des membres universitaires du jury face à sa tenue vestimentaire, sa manière de se déplacer dans la pièce, son réflexe de leur serrer la main montrent combien des attributs liés à l'hexis corporelle ${ }^{6}$ interviennent comme indicateurs de distance avec les codes universitaires.

Les membres universitaires du jury attendent implicitement une posture difficile à définir : un ton de neutralité impliquée, une distance respectueuse, le rejet d'un comportement trop scolaire et la valorisation d'une attitude de liberté intellectuelle. Trop d'assurance et le jury pense que le candidat n'a pas la bonne appréciation du niveau attendu et la distance suffisante vis-à-vis de la communauté scientifique. Cet implicite participe à la décision selon des codes comportementaux, linguistiques et intellectuels (Boudieu et Passeron, 1964).

6. Bourdieu P. (1964) définit l'hexis corporelle comme est un ensemble de dispositions pratiques corporelles, manières de se tenir, de parler, socialement construites. 


\subsubsection{Le dossier}

Le dossier est une mise en scène et le jury statue sur une présentation adaptée, comme dans cet exemple : une jeune femme avait échoué plusieurs fois à une licence de lettres modernes, élément discriminant en règle générale. Employée dans un centre d'appels, elle ne pouvait pas justifier d'acquis formels mais seulement d'un contrat de quelques mois dans une librairie pour la jeunesse dans une fonction de gestion des commandes, et d'un profil de lectrice capable d'analyse littéraire. Elle a su exposer sa maittrise de la littérature de jeunesse, démontrant son niveau universitaire et le jury lui a accordé la licence. Il n'a pas statué en soupesant uniquement l'expérience professionnelle au regard d'un contenu d'un diplôme, mais il a pourtant fait son travail : relever dans la référence à ses activités professionnelles les traces d'acquis académiques comparables à ceux attendus des diplômés.

Un dossier long et bien présenté est une preuve de l'implication du candidat, de la "valeur » qu'il reconnait au diplôme, par exemple pour le jury de sciences du sport : «Bon, le dossier... complet, il y a du travail..." (professeur de mécanique humaine, membre permanent). Cet élément informe sur le temps consacré au travail de VAE et certains jurys statuent en fonction de cette " morale de l'effort ". C'est à la fois un indicateur de rigueur mais aussi d'une volonté de "séduire » le jury, de le considérer comme un autrui de valeur.

Les membres universitaires du jury cherchent des traces d'un habitus universitaire : un bon usage des termes scientifiques et de la bibliographie. Le candidat doit éviter le registre anecdotique et démontrer un esprit de synthèse. Les professionnels cherchent une pluralité dans les techniques convoquées : il est bon de ne pas désigner un « outil » comme unique solution. "La méthode X permet de tester... ", dit le candidat. "Oui mais il y a en a d'autres! ", l'interrompt un membre du jury de licence professionnelle "Qualité ", échangeant des regards avec ses voisins (professionnel, membre non permanent).

Enfin, dans la littérature professionnelle, le dossier de VAE fait référence au récit de vie (Laine A., 2009, p. 155) ${ }^{7}$ comme processus formateur et manière de restaurer des souvenirs pour reconstituer une analyse de ses compétences, ce qui interroge sur la nature des objets réellement évalués : la biographie professionnelle comme élément objectivé ? Une méta-capacité à mettre en mots une vie dans un récit agréable ? Ou encore l'effort de réflexivité comme outil de recul sur sa pratique ? Un récit trop descriptif dans lequel le candidat n’a pas opéré de tri entre les éléments va être moins bien évalué qu'un récit plus stratégique, comme pour ce jury de lettres et langues : "Il sait se mettre en valeur " (professeur d'anglais, membre permanent) ; "Il a déjà la démarche scientifique ", "en fait, on leur demande de problématiser leur expérience " (maitre de conférences en sciences de gestion, membre permanent).

7. Lainé A., « Histoire de vie ", in Boutinet J.P., , L'ABC de la VAE, Eres «Éducation - Formation », 2009, pp. 154-155. 
Produire un bon dossier, c'est connaître son lecteur et les savoirs qu'il valorise, c'est élaborer une stratégie pour le satisfaire : autant de compétences transversales attendues en VAE, formation initiale et continue.

\section{U. Une décision collective fondée 3 sur la division du travail de définition}

Ces observations signalent la présence d'une norme partagée de validation. Elle introduit un résultat surprenant dans un dispositif présenté comme difficile à assurer : nous observons qu'un consensus s'exprime dans la quasi-totalité des jurys. En dehors de quelques débats en 2002 autour du principe de la VAE, aucun jury ne manifeste de désaccords fondamentaux, les discordes se jouent à la marge sur des niveaux de validation partielle.

Plusieurs éléments permettent d'expliquer cette situation : la progressive professionnalisation de l'enseignement supérieur depuis les années 80 rapproche les cultures académiques et professionnelles. Création des diplômes professionnels, généralisation des stages, relations avec des intervenants professionnels : une affiliation mutuelle se manifeste dans un certain nombre de formations. Aujourd'hui, par exemple, un enseignant-chercheur en langue étrangère partage un certain nombre de codes académiques et professionnels avec un traducteur en maison d'édition.

Néanmoins, l'accord entre les définitions de la situation projetées par les participants est fondé sur une "division du travail de défnition"(Goffman, 1973, p. 18). Chacun est autorisé à proposer la version officielle des questions qui le concernent. Une règle implicite intervient dans les jurys : chaque catégorie de membre est porteuse d'un rôle qui lui attribue le droit de juger. Une distinction s'opère entre les enseignants-chercheurs et les représentants des métiers.

Pour les questions de contenu des expériences, le représentant d'un champ professionnel est autorisé à imposer des affirmations sans qu'elles ne soient, à aucun moment, remises en question. En jury de licence "Banque Assurance ", un directeur d'agence est, par exemple, le seul habilité à traduire en niveau de responsabilité et en diplôme universitaire, la description de formations professionnelles spécifiques aux carrières bancaires : " un vrai parcours de formation, s'il a obtenu ce titre ça révèle derrière, oui, des fonctions d'encadrement et d'analyse des produits, vraiment." (professionnel, membre permanent).

Les membres universitaires, en particulier ceux du noyau permanent, sont légitimes pour s'exprimer sur les compétences transversales, preuves d'un niveau académique : "quand on peut répondre à des questions sans parler de détail, logistique, matériel... sans s'attacher à une situation particulière mais au contraire globaliser, avoir une approche générale, parler et mettre en rapport plusieurs fonctions". (Entretien avec un maître de conférences en sciences de gestion, membre permanent). 
Les enseignants invités en fonction des spécialités du diplôme sont autorisés à participer à la composante disciplinaire de la décision. Le caractère indiscutable de leur position est très visible pour les diplômes de langues. Ils opèrent un tri dans les acquis du candidat, ses voyages, ses lectures et distinguent ce qui est de l'ordre de la culture générale, de la connaissance linguistique, des usages et de la civilisation d'un pays.

Enfin, le président du jury reste le garant du diplôme, de la cohérence entre le dispositif de VAE et les valeurs de l'université. En cas de doute, il tranche et rappelle la législation : "depuis le début, nous le disons, nous aurons les mêmes exigences que pour les étudiants!" (professeur en anglais, membre permanent).

\section{Conclusion}

La décision est le fruit d'une représentation de la situation, construite collectivement à l'articulation des cultures professionnelles et académiques. L'activité des jurys, entre bricolage, construction d'une norme, affiliation progressive, permet d'atteindre un accord autour de preuves partagées. Dans cette construction, même si les éléments d'expérience professionnelle forment l'ossature de la décision, un attribut qu'on peut désigner sous le terme d'habitus universitaire est fondateur d'une VAE réussie.

Une présentation de soi correspondant aux codes culturels de l'enseignement supérieur est la condition minimale d'une validation satisfaisante. Réussir une VAE, c'est être capable d'intérioriser les règles sociales implicites de l'université correspondant aux allants-de-soi décrits par Coulon (1997) : savoir se comporter face au jury, trouver les codes de communication à l'oral comme à l'écrit. En VAE, cela se manifeste à travers la capacité à choisir dans son parcours les éléments significatifs, à lire les attentes implicites d'un référentiel. Cette mise en scène, à travers un entretien et un dossier, permet au jury de se forger une représentation de la situation, faisant appel à des éléments du passé, des stéréotypes, une mémoire plus ou moins reconstituée des décisions précédentes. Des éléments de culture (académique, professionnelle), à connotation morale (usurpation du rôle, sincérité), voire même liés aux signes non verbaux, (habillement, manière de se tenir) interviennent dans le jugement, comme pour toute autre situation d'interactions.

Nos résultats incitent à ouvrir la réflexion sur le rôle de l'accompagnateur, ce qui mériterait un travail approfondi. En effet, il semble que la réussite de cette mise en scène se nourrisse des conseils de l'accompagnateur, s'il a lui-même intériorisé les codes universitaires. Il guide les candidats vers le référentiel caché dans un travail d'affiliation progressive avec la norme universitaire. "C'est paradoxal! Il faut à la fois que le candidat montre sa créativité, son autonomie, puisqu'on est à l'université, mais on lui demande de remplir un dossier très cadré, projet, CV..., finalement on le cadre pour quil plaise au jury, quil soit académique " (Conseillère VAE, ingénieur d'études).

Le parcours formel d'accompagnement est un chemin normatif dans l'objectif de présenter au jury des candidats acceptables. Dans l'université étudiée, les accompagnateurs 
rencontrent régulièrement les responsables de formation pour s'imprégner des éléments implicites des diplômes et les transmettent à leur tour aux candidats. Nous pouvons faire l'hypothèse que leur présence en jury favorise une intériorisation des éléments de jugement, leur permettant de mettre en scène leur candidat d'une manière adéquate. Ces deux facteurs, binôme accompagnateur/enseignant-chercheur et présence en jury sont peutêtre des éléments de compréhension des taux de réussite satisfaisants dans l'université présentée.

\section{Bibliographie}

Auras E. (2011), De paradoxes en compromis, l'intégration de la formation continue à l'université? Thèse de troisième cycle, Poitiers,.

Bellini S. (2007), "L'expérience de la validation » in Neyrat Frédéric (dir.), La validation des acquis de l'expérience, la reconnaissance d'un nouveau droit, Editions du Croquant.

Bonicco C. (2006-07), " Goffman et l'ordre de l'interaction, un exemple de sociologie compréhensive ", Philonsorbonne 48/129 n 1, p. 31.

Bourdieu P., Passeron J-.C. (1964), Les Héritiers, les étudiants et la culture, Paris, Minuit.

Bourdieu P. (1993), (dir), La misère du Monde, coll. « Points, » Editions du Seuil, Paris.

Coulon A. (1997), Le métier d'étudiant, L'entrée dans la vie universitaire, PUF, Paris.

Diaz F. (2005), « L’observation participante comme outil de compréhension du champ de la sécurité » http://champpenal.revues.org/document79.html.

Feutrie M. (2004), "Une autre évaluation, une autre validation pour l'expérience ", Education Permanente, $\mathrm{n}^{\circ} 158$, pp. 99-114.

Gehin J.-P. (2011), «Les dossiers de VAE ; place des acquis de l'engagement » in Auras E., Géhin J.-P., (dir.), La VAE à l'université, approche monographique, Rennes, PUR, p. 115.

Goffman E. (1973), La mise en scène de la vie quotidienne, 1- la représentation de soi, Les Editions de Minuit, Paris.

Laine A. (2009), « Histoire de vie » in Boutinet J.-P., L'ABC de la VAE, ERES « Education - Formation », p. 154.

Mayen P., Mayeux C. (2009), « Regards sur l'activité de jurys VAE / des prescriptions du dispositif aux pratiques en séance», in Mayen P. et Savoyant A., Élaboration et réduction de l'expérience dans la validation des acquis de l'expérience, ReliefCereq n 28, p. 13.

Mayen P., Tourneur C. (2009), "Les jurys ont-ils quelque chose à apprendre ", in Mayen P. et Savoyant A. Élaboration et réduction de l'expérience dans la validation des acquis de l'expérience, Relief Cereq $\mathrm{n}^{\circ}$ 28, pp. 59-67. 
Metral J.-F., Mayen P. (2009), "Les difficultés de la validation des acquis de l'expérience », in Mayen P. et Savoyant A., Élaboration et réduction de l'expérience dans la validation des acquis de l'expérience, Relief Cereq $\mathrm{n}^{\circ} 28$, p. 29.

Mencacci N., Vial M. (2004), «Valider les acquis de l'expérience à l'université, une occasion de prendre en considération les savoirs informels ", Éducation permanente n 159.

Prot B. (2003), «Analyse du travail des jurys en validation des acquis : l'usage du référentiel ", L'orientation scolaire et professionnelle, $\mathrm{n}^{\circ} 32 / 2$, p. 219.

Ropé F. (2005), "La VAE à l'Université française : entre savoirs et expérience », Cahiers de la recherche sur l'éducation et les savoirs, $\mathrm{n}^{\circ} 4$.

Soulé B. (2007), «Observation participante ou participation observante ? Usages et justifications de la notion de participation observante en sciences sociales ", Revue recherches qualitatives, vol. $27, \mathrm{n}^{\circ} 1$.

Watson F.R. (1999), "Le travail de l'incongruité ", in le parler frais de Erving Goffman, Paris, Minuit, p. 83. 


\section{École, alphabétisation et lutte contre lillettrisme}

Hommage à Hocine Khelfaovi

Le comité de rédaction des CRES

DOSSIER

Introduction: Analphabétisme et illettrisme au Nord et au Sud. Quelles voies pour la recherche en sciences sociales et la construction de politiques publiques?

Éric Lanove

La mesure de l'analphabétisme en question. Le cas de l'Afrique subsaharienne

Alain Mingat, Francis Ndem et Adeline Seurat

Prévenir l'illettrisme dès l'école primaire : analyse du cas français à la lumière de

la comparaison internationale

Caroline Viriot-Goeldel

Retour sur l'illettrisme en France : malaise politique, controverse scientifique, complément méthodologique

Agnès Villechaise et Joël Zaffran

Évangélisation et alphabétisation au Togo sous domination coloniale (1884-1960) Koffi Nutefé Tsigbé

Alphabétisation et éducation en langues nationales dans les politiques globales de

l'éducation au Bénin : valeurs, principes d'actions et stratégies d'acteurs

Abdel Rahamane Baba-Moussa

L'expérience d'éducation zapatiste au Chiapas : entre pratiques politiques et

imaginaires autochtones à l'école

Bruno Baronnet

La littératie pulaar : nationalisme culturel, mouvement social ou instrument du développement? Marie-Ève Humery

De l'utopie à la prescription de normes : les investissements politiques liés à l'apprentissage

de la lecture

Sandrine Garcia

Les analphabètes au Maroc : un groupe homogène en demande d'alphabétisation? Sophie Cerbelle

Écritures de femmes déclarant ne pas écrire. Quatre femmes latino-américaines à Paris Delphine Leroy

Représentations de l'écrit et image de soi : les obstacles à l'apprentissage.

Un retour sur expérience

Anne Torunczyk

HORS-THÈME

Héritage colonial et appropriation du «pouvoir d'éduquer ».

Approche socio-historique du champ de l'éducation primaire en RDC

Géraldine André et Marc Poncelet

Excellence sociale ou excellence scolaire? Les modes d'admission dans les écoles d'élite irlandaises Aline Courtois

Écoles franco-arabes publiques et daaras modernes au Sénégal : hybridation des ordres normatifs concernant l'éducation

Sophie D'Aoust

Revue internationale de sciences sociales éditée par l'ARES, avec le soutien des Éditions de la Maison des Sciences de l'Homme/Paris, de l'IRD et du CURAPP ARES : IRD - 32, avenue Henri Varagnat - 93143 Bondy cedex - France

Distribution en librairie : FMSH-Diffusion - 18 rue Robert Schuman, CS 90003,94227 Charenton-le-Pont Cedex

Pour les articles en ligne voir le site : cres.revues.org Pour l'achat en ligne des numéros voir le site : www.lcdpu.fr Pour l'achat par chèque (à l'ordre de l'ARES) commander à : revue.cres@yahoo.fr Prix unitaire : 20 euros 